\title{
Pulsed-assisted escape from zero voltage state in Josephson systems.
}

\author{
G. Rotoli ${ }^{\mathrm{a}, \mathrm{b}}$, C. De Leo ${ }^{\mathrm{a}}$, G.P.Pepe ${ }^{\mathrm{c}}$, L.Parlato ${ }^{\mathrm{d}}$, G.Peluso ${ }^{\mathrm{c}}$.
}

${ }^{a}$ Dipartimento di Energetica, Università dell'Aquila, L'Aquila, Italy.

${ }^{b}$ I.N.F.M. , UdR Università dell'Aquila, L'Aquila, Italy.

${ }^{c}$ I.N.F.M.-Dipartimento Scienze Fisiche, Università di Napoli Federico II, Naples, Italy.

${ }^{d}$ Seconda Università degli Studi di Napoli, Facoltà di Ingegneria, Aversa ( CE ), Italy.

\begin{abstract}
The behavior of a Josephson system under pulsed operation is of utmost importance for developing reliable digital devices working at very high clock frequencies. Information about effect of thermal noise over pulsed operation is also useful to design errors free devices. Recent experiments in a system of two stacked junctions show that a pulsed operation on the first junction (injector) of the stack drive the Josephson biased second junction (detector) into the resistive state. The experiment was interpreted as pulsed-assisted escape toward resistive state as a consequence of an excitation of large non-linear oscillations in the detector junction that, with the contribution of thermal noise, drives out the junction from the zero voltage state. Moreover, in the same experiment for an unstability of the resistive state toward the zero voltage state under the pulsed operation was also noted. By means of a systematic numerical approach to the problem, we present a study of the pulsed-assisted escape using the framework of the thermal escape theory for the direct transition from zero voltage state to resistive state. We study the single junction case and a stacked configuration as examples of different systems showing pulsed-assisted escape.
\end{abstract}

Keywords: superconducting devices, pulsed operation, noise, numerical simulations.

Corresponding author: G.Rotoli, Dipartimento di Energetica, Università di L'Aquila, Località Monteluco, Roio Poggio I67040, L'Aquila.

Fax: +0862-434303

E-mail: rotoli@ing.univaq.it 
Non-equilibrium phenomena in Josephson junctions are among the most promising candidates for novel applications of superconducting electronics. Transistor-like devices [1], integration with fast optoelectronic devices [2,3], and finally bi-stable devices [4] have been recently developed. In this paper we present some numerical results concerning the behavior of a current biased Josephson Junction (JJ) under the influence of an electronic current pulse injected both directly in the junction via its bias current and from a second JJ forming a double tunnel junction stacked device. We study the passage from the zero voltage state to the dissipative state produced by injected electronic pulses, and the possibility of resetting, i.e., the pulse assisted return to zero voltage, under suitable conditions for injected electronic pulses in terms of their amplitudes and bias currents. Comparison with a recent experiment [4] on a stacked device is also shown.

The numerical results have been obtained by a numerical approach based on the Perturbed SineGordon Equation (PSGE) for the superconducting phase difference $\varphi$ which in normalized units reads:

$$
-\partial \varphi_{x x}+\partial \varphi_{t t}+\alpha \partial \varphi_{t}+\sin \varphi=\gamma_{B}+\gamma_{N}(x, t)+\gamma_{P}(x, t)
$$

here time is normalized to plasma frequency $\omega_{\mathrm{J}}$ and space to Josephson length $\lambda_{\mathrm{J}}$; the parameter $\alpha$ represents the losses and $\gamma_{B}$ is a constant bias current. In Eq.(1) $\gamma_{N}(x, t)$ is a Gaussian white noise at $4.2 \mathrm{~K}$ modeled as in Ref.[5]. A pulse train is applied to the junction biased on the zero voltage state via the term [4]:

$$
\gamma_{P}(x, t)= \begin{cases}2 \Gamma\left(\frac{x}{l}\right)\left(\frac{t}{T}\right) & \text { for } 0 \leq t \leq \frac{T}{2} \\ 2 \Gamma\left(\frac{x}{l}\right)\left(1-\frac{t}{T}\right) & \text { for } \frac{T}{2} \leq t \leq T \\ 0 & \text { for } T \leq t \leq T_{p}\end{cases}
$$

where $T$ is the pulse length, $l$ the normalized junction length and $T_{p}$ the time interval between pulses. The pulse function is linear in space and has the form of a triangular pulse train in time.

Results for single junction with a normalized length, $l=1$ and $\alpha=0.15$ are shown in Fig. 1 where we report the average spatial voltage in the junctions. A train of pulses of normalized time length $T$ equal to 100 separated by $T_{\mathrm{p}}=1500$ was applied to the junction. Peak amplitude was set to $\Gamma=-20$ (the minus sign is essential for obtain the reset of the junction). Temperature was set to $4.2 \mathrm{~K}$ and critical current to $1 \mu \mathrm{A}$. From Fig. 1 is evident that increase of bias increases the escape rate $\Pi$ (here defined simply as the inverse 
of the mean lifetime in the zero voltage state). For the large bias values a saturation effect take place, i.e., in many cases the junction remains in the resistive state also after the resetting pulse. Similar considerations apply also to longest junctions (cf.[6]).

Improved results can be obtained using a stacked configuration in order to separate the pulse injection phase from junction response. A stacked configuration is also more interesting for applications being a natural three-terminal device. For the stacked junctions we pump one junction (injector) in the stack with a train of variable amplitude pulses and observing the effect on the other junction (detector). In stacked junctions coupling depends on the second spatial derivative of the phase [7], this justifies the use of a pulse modeled as in Eq.(2). In normalized units the PSGE's for injector, $\psi$, and detector, $\varphi$, are:

$$
\begin{aligned}
& \varepsilon \partial_{x x} \varphi-\partial_{x x} \psi+\partial_{t t} \psi+\alpha \partial_{t} \psi+\sin \psi+\gamma_{P}(x, t)+\gamma_{N}(x, t)=0 \\
& \varepsilon \partial_{x x} \psi-\partial_{x x} \varphi+\partial_{t t} \varphi+\alpha \partial_{t} \varphi+\sin \varphi+\gamma_{B}+\gamma_{N}(x, t)=0
\end{aligned}
$$

where $\varepsilon$ is the stack coupling, $\alpha$ the loss parameter [7]. The injector junction is not biased. We choose $l=1$ and $\alpha=0.15$ for both junctions and $\varepsilon=-0.85$ for the stack coupling. Here $\gamma_{\mathrm{B}}$ is the constant detector bias which is always larger than 0.195 sufficient to exclude the effect of thermal return current. Saturation take place also in stacked configuration but for larger values of the bias current $\left(\gamma_{B}>0.235\right)$. Results are reported in Fig. 2 for a positive pulse amplitude $\Gamma=20$ (here the pulse sign is inessential): from a) to c) the plots show the spatial average voltages for increasing (detector) bias current. For sufficiently large bias current the device has a flip-flop-type logic characteristic, i.e. it commutes for each pulse across the injector. Qualitatively similar results can be obtained also with different values of the stack coupling, pulse length, spatial dependence and by changing the pulse amplitude $[4,6]$.

Simulations show that increasing the temperature will smear the set on of transition to resistive state in bias current in both single and stacked configurations. This is very reminiscent of thermal escape from zero voltage $[5,8]$. Pulses increase the energy of the Josephson oscillations causing a thermal escape toward resistive state. If this hypothesis is true the escape rate would depends exponentially on bias current and temperature, i.e., $\Pi=\left(\omega_{\mathrm{J}} / 2 \pi\right) \exp \left(-\mathrm{U}_{0}\left(\gamma-\gamma_{0}\right)^{3 / 2} / \mathrm{kT}\right)$, where $\mathrm{U}_{0}$ and $\gamma_{0}$ are unknown parameters to be fitted to the data $[5,8]$. The results of the analysis of escape rate are reported in Fig. 3a for the single junction with $1=1$ : the behavior of the escape rate fits with good approximation the theoretical prediction of linearity. Fitting of free parameters give $\gamma_{0}$ larger than 0.6 and $\mathrm{U}_{0}$ roughly of $0.2 \%$ of Josephson energy. The large value of $\gamma_{0}$ indicates that non-linearity and saturation effects present in single junction case will compete with thermal activation giving an unexpected large transition region. The analysis of escape rate 
in the stacked configuration is reported in Fig.3b: the fit to linear dependence of $(\log \Pi)^{3 / 2}$ of numerical data is very good and we find $\gamma_{0}=0.267$ and $\mathrm{U}_{0}$ about $2 \%$ of Josephson energy. The again (relatively) large value of $\gamma_{0}$ indicates that saturation is present also in stacked configuration. We underline that the complex non-equilibrium phenomena taking place in the junctions subject to the pulsed operation could easily hinder any evidence of a thermal regime, so it is somewhat surprising that the simple model derived for thermal escape in short junctions will give a good fit to the numerical data. Finally we note that in principle the same analysis could be applied also to the back-switching transition. We think that the return current phenomenon [9] is important in order to determine the reset to the zero voltage state, but the analysis is more difficult.

Stacked tunnel JJs have been fabricated by following a novel process developed for high-quality three terminals superconducting electronics [10]. The process and the junction characterization have been described in ref.[4]. The Josephson penetration depth $\lambda_{\mathrm{J}}$ was estimated to be $70 \mu \mathrm{m}$, thus implying a ratio $\mathrm{L} / \lambda_{\mathrm{J}} \approx 1.4$ for a Josephson critical current $\mathrm{J}_{\mathrm{c}}=80 \mathrm{~A} / \mathrm{cm}^{2}$ and $\mathrm{L}=100 \mu \mathrm{m}$. The bottom junction was used as injector of current pulses, whose effect was observed across the top junction (detector). The injected pulses had rise-times of $2 \mathrm{~ns}, 600 \mu$ s of duration, delay time $20 \mathrm{~ms}$. They were supplied by a conventional pulse generator (EG\&G Mod. 480). Each pulse was split and sent simultaneously to both the injector junction and to a digital oscilloscope (Le Croy Mod. 9361, 300MHz) for triggering the waveforms acquisition. With the detector biased at $\mathrm{I}<\mathrm{Ic}$, the output voltage across the detector was measured by a standard PAR Mod. 5113 pre-amplifier (300 kHz BW). In Fig.4 we report the output detector voltage for different injection pulse amplitudes. Apart some reflections in the signal due to a not completely matched injection line, a clear correlation between injected pulses and the detector voltage is present. In particular, in the presence of injected pulses across the bottom junction, a change of the detector voltage from the state $\mathrm{V}=0$ to the quasi-particle branch of the I-V curve and viceversa is observed similar to that described above in the simulations (see Fig.2 and ref.[4]). This behavior, observed both in single shot and a.c. steady-state measurements (-the delay time between pulses was $20 \mathrm{~ms}-$ ), cannot be ascribed to the specific loading of the detector junction (-the load line remains the same during the switching measurements-). For larger amplitudes no back-switching is observed, i.e., a saturation phenomenon similar to that observed in bias in the simulations.

In conclusion, we presented numerical simulations and measurements of pulse activated transitions from the metastable to the running state and viceversa in both single junction and stacked Josephson 
systems. At least for the stacked configuration where the fitting give realistic parameters, an interval of bias currents exists (here from 0.2 to 0.235 for the detector bias current) where there is evidence of a thermal regime, i.e., the numerically evaluated escape rate can be fitted by the simple short junction theoretical thermal escape rate. Experimental results on stacked device compare favorable with numerical simulations of stacked junctions model.

We are grateful to G.Filatrella, C.Granata, E.Esposito, M.Russo, M.Valentino and A.Barone for useful discussions and highlights. This work is supported by the I-MURST COFIN2000 Program Dynamics and Thermodynamics of vortex structures in supeconducting tunneling. 


\section{REFERENCES}

1 G.P.Pepe, G.Ammendola, G.Peluso, A.Barone, E.Esposito, R.Monaco, N.E.Booth, Appl.Phys.Lett. 77 (2000) 447.

2 K.S.Il'in, I.Milostnaya, A.A.Verevkin, G.N.Gol'tsman, E.M.Gershenzon, R.Sobolewski, Appl.Phys.Lett.73 (1998) 3938.

$3 \quad$ R.Sobolewski and J.R.Park, IEEE Trans.Appl.Supercond.11 (2001) 727.

4 G.P. Pepe, G. Peluso, M. Valentino, A. Barone, L.Parlato, E. Esposito, C. Granata, M. Russo, C. De Leo and G. Rotoli, accepted for the publication on Appl.Phys.Lett, arXiv: cond-mat/0105342

5 M.G.Castellano, G.Torrioli, C.Cosmelli, A.Costantini, F.Chiarello, P.Carelli, G.Rotoli, M.Cirillo, R.L.Kautz, Phys.Rev.B 54 (1996) 15417.

6 G.Rotoli, C.De Leo, G.P.Pepe, G.Peluso, M.Valentino, A.Barone, L.Parlato, poster presented to EUCAS-2001, 26-30 August 2001; see http://ing.univaq.it/energeti/research/Fisica/supgru.htm

7 A.V.Ustinov, H.Kohlstedt, M.Cirillo, N.F.Pedersen, G.Hallmanns, C.Heiden, Phys.Rev.B48 (1993) 10614; S. Sakai, P. Bodin and N. F. Pedersen, J.Appl.Phys.73 (1993) 2411.

8 M.H.Devoret, J.M.Martinis, D.Esteve, J.Clarke, Phys.Rev.Lett.53 (1984) 1260.

9 M.G.Castellano, G.Torrioli, F.Chiarello, C.Cosmelli, P.Carelli, J.Appl.Phys.86 (1999) 6405.

10 G.P.Pepe, G.Peluso, R.Scaldaferri, L.Parlato, C.Granata, E.Esposito and M.Russo, 2001 accepted for publication on Eur. Phys. Journ 


\section{FIGURE CAPTIONS :}

Fig.1: Effect of electronic pulse injection on the single junction l=1: the plots show the average voltage in the junction subject to a pulse train with $T=100$ n.u., separated by $T p=1500$ : (a) $\gamma_{B}=0.21$; (b) $\gamma_{B}=0.25$; (c) $\gamma_{\mathrm{B}}=0.35$. Time and voltage are normalized to $1 / \omega_{\mathrm{J}}$ and $\omega_{\mathrm{J}} \Phi_{0}$ respectively. Negative part of pulse injection is not shown.

Fig.2: Simulated flip-flop transition of two junction stack vs the detector junction bias current: (a) $\gamma_{B}=0.205$; (b) $\gamma_{B}=0.21$; (c) $\gamma_{B}=0.22$. The dotted curve with squares refers to injector junction voltage (not in scale), the circles curve to detector junction voltage. Time and voltage are normalized to $1 / \omega_{\mathrm{J}}$ and $\omega_{J}$ $\Phi_{0}$ respectively. Injector curve is scaled by a factor 10 .

Fig.3: Calculated logarithmic escape rates: (a) single junction; (b) stacked configuration. In both case parameters of junctions are $l=1, \alpha=0.15$. Average is calculated on about 200 to 600 lifetimes depending on bias current.

Fig.4: Experimental results: the detector junction output voltage as a function of injected electronic pulses across the coupled junction. The scale is referred to the detector output voltage, while pulses are reported in arbitrary units. 


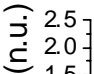

(a)
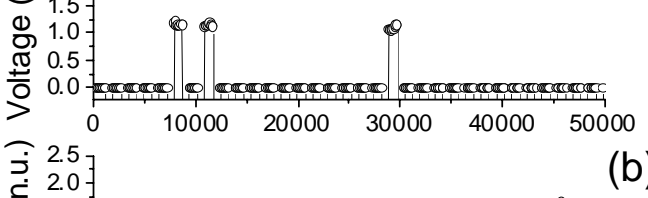

다.

ठ) $1.0=$

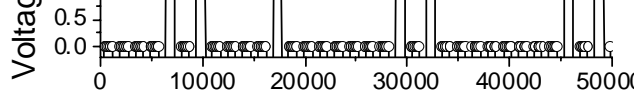

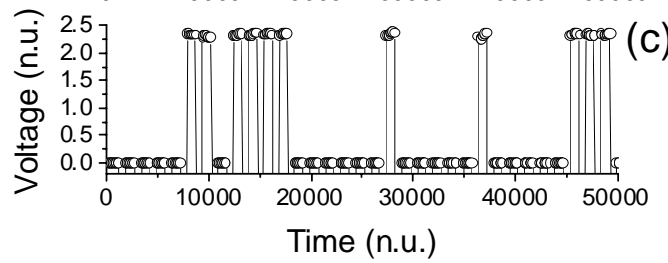

(b)

(c) 


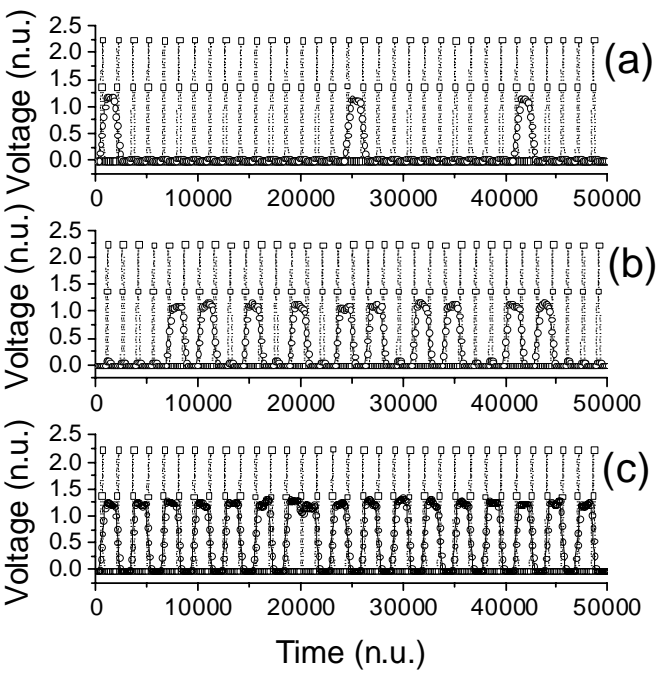



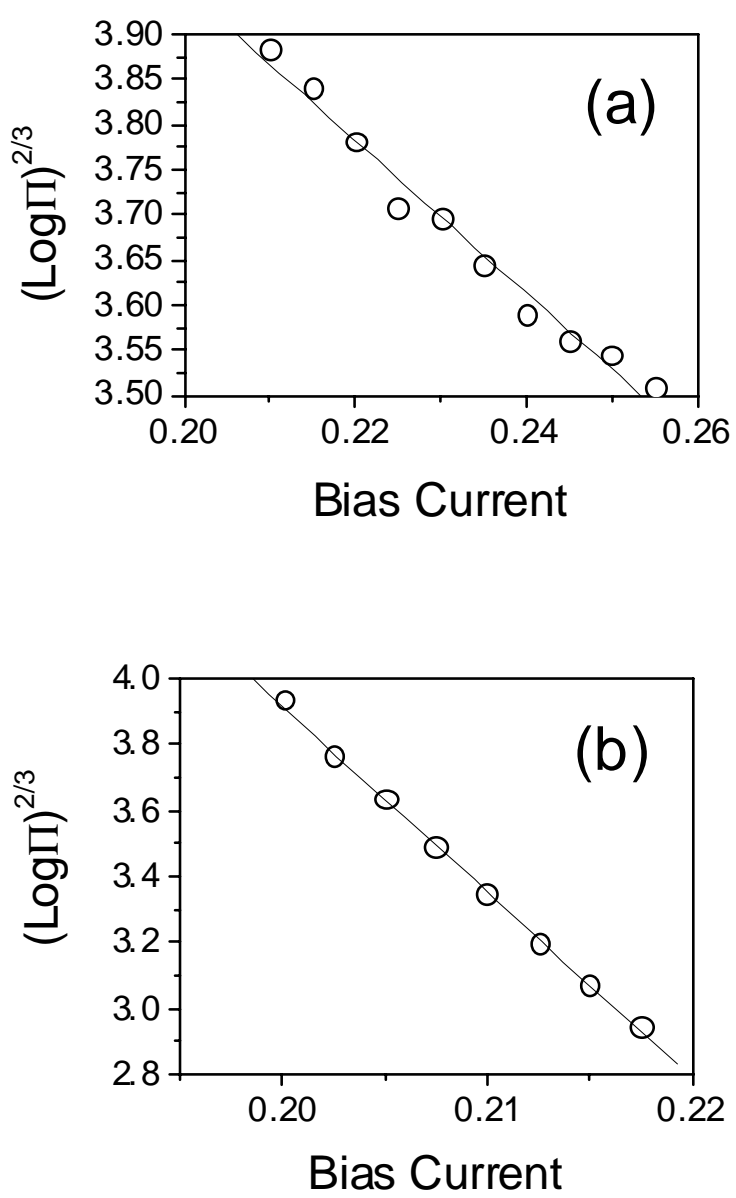


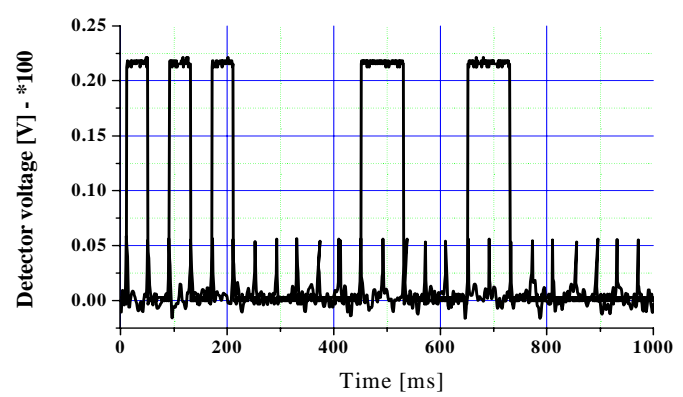

